Document downloaded from:

http://hdl.handle.net/10251/46512

This paper must be cited as:

Eugenio Pellicer; Yepes Piqueras, V.; Correa Becerra, CL.; Alarcon, L. (2014). MODELO PARA LA INNOVACIÓN SISTEMÁTICA EN EMPRESAS CONSTRUCTORAS. Journal of Construction Engineering and Management. 140(4):40140011-40140018. doi:10.1061/(ASCE)CO.1943-7862.0000468.

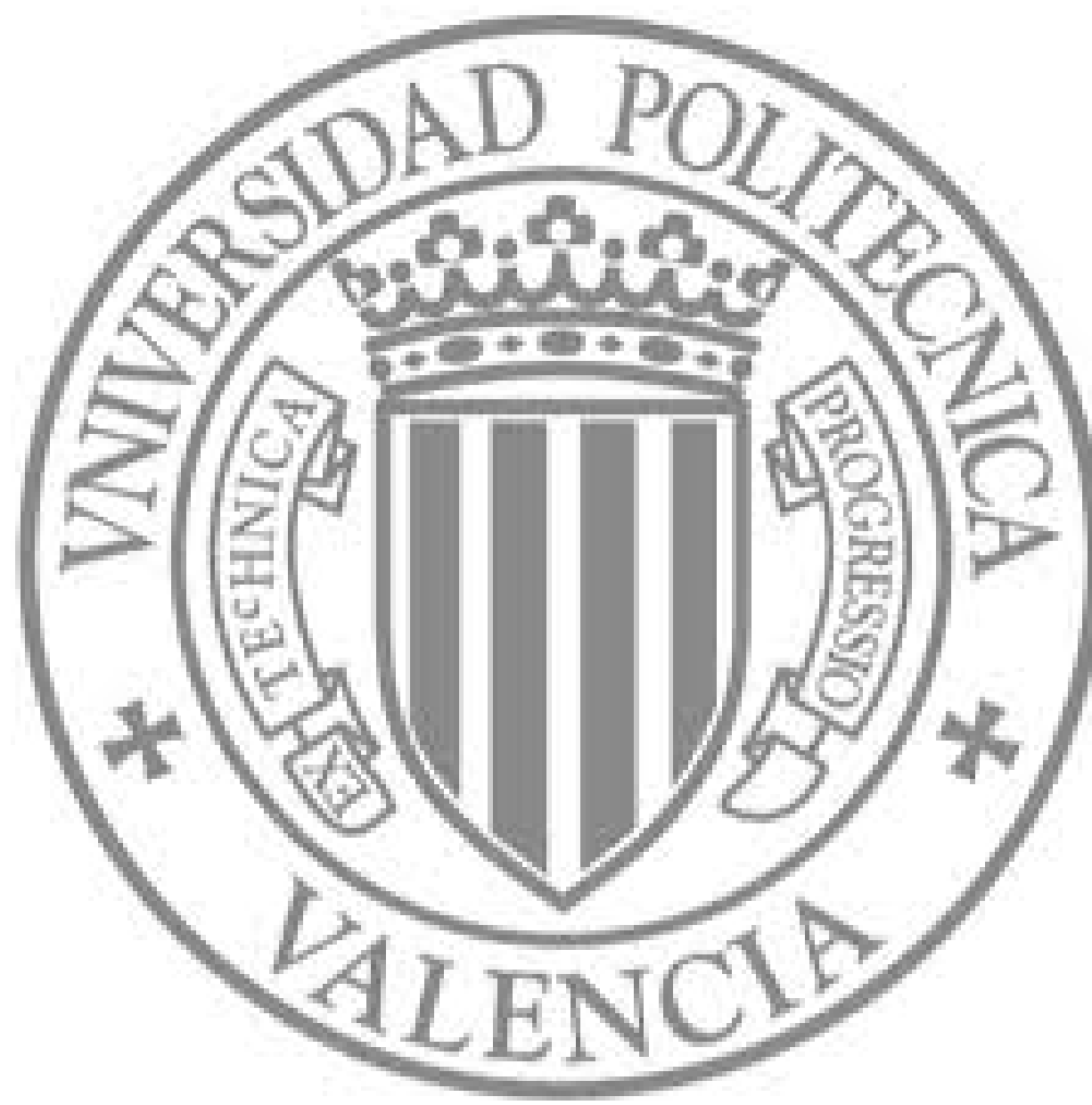

The final publication is available at

http://dx.doi.org/10.1061/(ASCE)CO.1943-7862.0000468

Copyright American Society of Civil Engineers 


\section{MODELO PARA LA INNOVACIÓN SISTEMÁTICA EN EMPRESAS}

\section{CONSTRUCTORAS}

\section{Eugenio Pellicer ${ }^{1}$, Víctor Yepes ${ }^{2}$, Christian L. Correa ${ }^{3}$ y Luis F. Alarcón ${ }^{4}$}

${ }^{1}$ Titular de Universidad (Associate Professor), ETSI Caminos, Canales y Puertos (School of Civil Engineering), Universidad Politécnica de Valencia, Camino de Vera s/n, 46022 Valencia, España, teléfono: +34.963.879.562, fax: +34.963.877.569, correo electrónico: pellicer@upv.es

${ }^{2}$ Titular de Universidad (Associate Professor), ICITECH, Universidad Politécnica de Valencia, Camino de Vera s/n, 46022 Valencia, España, correo electrónico: vyepesp@upv.es ${ }^{3}$ Instructor (Assistant Professor), Facultad de Ciencias de la Ingeniería (Faculty of Engineering Sciences), Universidad Católica del Maule, San Miguel 3605, Talca, Chile, correo electrónico: clcorrea@ucm.cl

${ }^{4}$ Profesor Titular (Professor), GEPUC, Pontificia Universidad Católica de Chile, Vicuña Mackenna 4860, Santiago, Chile, correo electrónico: lalarcon@ing.puc.cl

\section{RESUMEN}

Los motivos que impulsan a las empresas constructoras a innovar, así como los procedimientos que utilizan, no han sido suficientemente explorados hasta el momento en la literatura sobre gestión de la construcción. Este artículo describe los “cómos” y “por qués” que respaldan el impulso innovador en una empresa constructora. El método de investigación se fundamenta en la validación de un modelo desarrollado mediante un estudio de caso; éste 
se centra en una empresa constructora de tamaño medio que implementó y certificó un sistema de gestión de la innovación, según lo establecido en la norma española UNE 166002. Los estudios desarrollados por los autores durante cinco años generaron un conjunto de 18 proposiciones que definen un modelo explicativo de la gestión de la innovación. Este artículo reporta la validación externa del modelo por medio de un conjunto de entrevistas, cuyos resultados corroboran plenamente 15 de las proposiciones planteadas. Los principales inductores de innovación en las empresas constructoras son los problemas técnicos que aparecen en obra, los requerimientos de los clientes y la alta dirección de la empresa. Las oportunidades de innovación se identifican como resultado del examen de los procesos internos de la empresa, de las obras y del entorno. Identificar, desarrollar y transferir una solución innovadora requiere la implantación de la vigilancia tecnológica y de la gestión del conocimiento en la organización. Finalmente, la investigación concluye que el principal beneficio de la gestión de la innovación es el incremento de la capacidad técnica de la empresa, mientras que las dos principales barreras para la innovación son la priorización de los procesos productivos y la falta de apoyo de los dirigentes de la empresa.

\section{PALABRAS CLAVE:}

Empresa Constructora - Gestión Empresarial - Innovación - Proceso - Normalización 


\section{INTRODUCCIÓN}

La gestión de la innovación en una empresa se lleva a cabo mediante un conjunto de actividades y de decisiones que incrementan el valor de los productos y de los servicios ofertados, tanto a los clientes externos como al resto de partes interesadas, o bien cumplen con otros objetivos estratégicos empresariales (Ko 2009, Trkman 2010). Este planteamiento considera la innovación como "la aplicación de ideas que son nuevas para la empresa, tanto si las nuevas ideas se materializan en productos, procesos, servicios, organización del trabajo, sistemas de gestión o de comercialización” (Gibbons et al. 1994). En este sentido, el objetivo final de un sistema de gestión de la innovación es fortalecer la competitividad de la empresa para que sobreviva a largo plazo (Evangelista et al. 1997). Sin embargo, esta relación entre innovación y competitividad aún no se percibe de forma nítida por parte de las empresas constructoras (Winch 1998, Harty 2008). Este motivo conduce a formular un modelo capaz de explicar el modo y las razones que impulsan la gestión de la innovación en una empresa constructora, y de hacer aflorar algunas barreras entorpecedoras para la adopción de estrategias de negocio innovadoras que permitan incrementar la competitividad en este tipo de organizaciones.

Las empresas modernas se gestionan basándose en procesos que tienden a transformar las organizaciones verticales, estructuradas según funciones, en otras de tipo horizontal, orientadas hacia actividades que aportan valor añadido al cliente (Vanhaverbeke y Torremans 1999). Estos procesos suelen ser continuos y, al menos parcialmente, recurrentes en sus actividades (Tidd et al. 1997, Gann 2000, Gann y Salter 2000); es por ello que las empresas desarrollan procedimientos para sistematizarlos y simplificarlos. Davenport (2005) justifica la normalización de los procesos señalando que éstos facilitan las comunicaciones internas y 
externas de las empresas; permiten también el intercambio entre los recursos, lo que flexibiliza y mejora la eficiencia del proceso, y la comparación entre distintas empresas (“benchmarking”). Las actuales tecnologías que soportan estos procesos empresariales favorecen la normalización y el intercambio de datos e información.

La gestión de la innovación se puede formular como un proceso empresarial, que además resulta crítico para la competitividad de una organización (Tidd et al. 1997, Vanhaverbeke y Torremans 1999). Es un proceso extremadamente complejo e incierto como consecuencia de su carácter evolutivo e interactivo (Veugelers y Cassiman 1999). Gann (2000) destaca las particularidades de las empresas que producen y gestionan por proyectos (la mayoría de las del sector de la construcción) en las que existen problemas adicionales de coordinación que afectan a la gestión del conocimiento en la organización e inhiben la capacidad innovadora de estas empresas. Algunos autores (Dulaimi 1995, Gann 2000, Gann y Salter 2000, Pellicer et al. 2008) indican que la innovación puede ser planificada, organizada, dirigida y controlada, también en el sector de la construcción, igual que cualquier otro proceso empresarial; sin embargo, la realidad es que muchas empresas que generan innovaciones lo hacen de forma puntual, alejadas de procesos de generación de ideas de forma metódica y continua.

Existen autores que señalan la escasa capacidad innovadora del sector de la construcción, altamente tradicional y ligado a las costumbres locales (Serpell 2001, Blayse y Manley 2004, Taylor y Levitt 2004). Otros trabajos abundan en el aspecto puntual de la innovación en la construcción (Winch 1998, Gann y Salter 2000). Los problemas que surgen en las obras provocan la generación de soluciones específicas o invenciones espontáneas (Nam y Tatum 1992). Este enfoque informal de la innovación no aprovecha las ventajas de su sistematización como un proceso que permite generar valor añadido a los clientes y a otras 
partes implicadas en el ciclo de vida de la infraestructura, tal y como lo plantea Manseau (1998).

Una vía que impulsa la innovación y que permite su sistematización, es la adopción de normas voluntarias, como la norma UNE 166002; UNE significa “Una Norma Española”, pudiéndose traducir al inglés como “A Spanish Standard”. La norma UNE 166002 se basa en un conjunto de subprocesos orientados a generar y documentar proyectos de innovación en la empresa; éstos subprocesos son: (a) vigilancia tecnológica, (b) creatividad, (c) planificación y ejecución de proyectos, (d) transferencia tecnológica, y (e) protección de resultados (AENOR 2006, Pellicer et al. 2008, Yepes et al. 2010, Mir y Casadesus 2011). La norma UNE 166002 sigue la filosofía de la mejora continua de los procesos, propia de la norma ISO 9001 relativa a la gestión de la calidad; además, el procedimiento para proporcionar certificados en innovación es muy similar al de la ISO 9001 (Pellicer et al. 2008). Algunos autores respaldan que una adecuada gestión de la calidad en la empresa mejora su capacidad innovadora (Perdomo-Ortiz et al. 2006 \& 2009). Casadesus et al. (2011) confirman que la aplicación conjunta de diferentes normas relativas a sistemas de gestión es beneficiosa para la empresa por las sinergias que genera. Las ventajas obtenidas recientemente por las empresas españolas al aplicar y certificar los sistemas de gestión de la innovación ha hecho que otros países como Portugal (norma NP 4457), Chile o México estén incorporando también versiones adaptadas de estas normas (Teixeira et al. 2009).

Este artículo presenta la fase final de una investigación de cinco años sobre gestión de la innovación llevada a cabo por los autores. El trabajo fue posible a raíz de la colaboración continua con una empresa constructora de tamaño medio, así como una cooperación puntual con otras empresas y profesionales del sector. Los trabajos previos comprendieron: (1) una 
profunda revisión bibliográfica y un establecimiento del marco teórico (Correa et al. 2007); (2) una presentación de la serie de normas UNE 166000, así como un estudio previo sobre la innovación en el sector de la construcción español (Pellicer et al. 2008); (3) el análisis estratégico de la empresa seleccionada como estudio de caso (Pellicer et al. 2010); (4) la implementación del sistema de gestión de la innovación en esa misma empresa (Yepes et al. 2010); y (5) la explicación del modelo derivado del estudio de caso (Pellicer et al. 2012).

El objetivo principal de este artículo es validar el modelo explicativo de la gestión de la innovación previamente desarrollado mediante el estudio de caso de una empresa constructora española; en el contexto de esta investigación se entiende la innovación tal y como la han definido Gibbons et al. (1994). La investigación aporta evidencias que permiten a las empresas constructoras entender la forma en que se desarrolla la innovación en sus organizaciones, los factores de los que depende y las barreras más importantes. De este modo, el artículo se organiza en cinco epígrafes adicionales. En primer lugar, se comentan las proposiciones obtenidas del modelo de gestión de la innovación construido previamente utilizando el estudio de caso de una empresa constructora. En segundo lugar, se describe el método de investigación utilizado para validar las proposiciones, y por tanto el modelo, que consiste básicamente en la realización de entrevistas a directivos encargados de la innovación en empresas ya certificadas, directivos de la empresa objeto de análisis y expertos externos. A continuación los resultados de la validación se muestran en un gráfico que muestra el grado de apoyo que obtiene cada una de las proposiciones. Los resultados se discuten en la siguiente sección, centrándose especialmente en el grado de discrepancia entre los grupos. El artículo finaliza indicando las principales conclusiones, limitaciones y líneas futuras de investigación. 


\section{MODELO PARA GESTIÓN DE LA INNOVACIÓN}

La gestión de la innovación comprende todas las actividades necesarias para la implantación eficiente de una idea en un producto o en un proceso que incremente la competitividad de una organización (Eaton 2001). Tidd et al. (1997) advierten que la gestión de la innovación debe entenderse como la generación de las condiciones necesarias dentro de una organización bajo la cual se llevan a cabo cambios tecnológicos, estratégicos u organizativos con una alta incertidumbre. Aplicada al sector de la construcción, esta gestión de la innovación puede implementarse a diferentes niveles, de mayor a menor amplitud (Correa et al. 2007): (a) en el sistema de investigación y desarrollo (I+D) nacional (Gann 1997); (b) dentro de la propia empresa (Gann y Salter 2000); (c) en proyectos o productos (Tatum 1987, Nam y Tatum 1992); y (d) durante el proceso constructivo (Kangari y Miyatake 1997).

El proceso de la gestión de la innovación en la empresa constructora ha sido objeto de estudio por parte de muchos autores. Manseau (1998) anima a la industria a adoptar una perspectiva sistémica y amplia con el fin de entender y expandir la innovación en la construcción. La mayoría las propuestas teóricas evalúan el éxito de la innovación en las empresas constructoras dependiendo de la respuesta adecuada a los factores ambientales e internos, tomando como referencia la teoría general de sistemas. A continuación se describen brevemente los modelos más destacados, extraídos de la literatura analizada.

Manseau (1998) y Seaden y Manseau (2001) proponen un modelo general aplicable a cada subsector pero centrado en la empresa. Este modelo considera el ciclo de vida completo de la infraestructura incluyendo a los restantes agentes y los diferentes tipos de interacciones entre 
ellos. Para Winch (1998), la innovación en las empresas constructoras nace de la relación existente entre las obras y las empresas. Gann y Salter (2000) desarrollan esta idea en un modelo en el que destacan seis dimensiones: empresas, cadena de suministro, proyectos, tecnología, regulación institucional y flujos de conocimiento. Seaden et al. (2003) proponen relacionar el entorno con la estrategia empresarial, afectando ambas a la capacidad innovadora de la organización. Sexton y Barrett (2003) definen un modelo basado tanto en el proceso de innovación como en el contexto interno y externo en el que ocurre. El contexto interno incluye la estrategia empresarial, el posicionamiento en el mercado, la organización del trabajo, la tecnología y los recursos humanos; el contexto externo comprende los diferentes entornos empresariales y sus interacciones. Dikmen et al. (2005) proponen un modelo sistemático para la innovación formado por cinco elementos básicos: objetivos, estrategias, inductores y barreras del entorno y, finalmente, factores organizativos.

El modelo explicativo de la gestión de la innovación en las empresas constructoras se presenta en la Figura 1, y está alineado con la propuesta de Seaden y Manseau (2001) sobre sistemas de conocimiento centrados en la empresa; según estos autores, la empresa es el centro de una red de suministradores, clientes, competidores y recursos. El modelo mostrado en la Figura 1 se explica con detalle y se discute desde el punto de su construcción teórica en Pellicer et al. (2012). Las empresas constructoras generan ideas novedosas que acaban transformándose en proyectos de innovación. Sin embargo, el éxito de este proceso descansa en una estrategia empresarial claramente alineada y dirigida hacia la generación de innovación. La estrategia sobre la que se apoya la innovación debe estar sólidamente fundamentada e integrada en el entorno de negocio, movilizando todas las capacidades organizativas de la empresa hacia la consecución de sus objetivos. Además, la estrategia debe incluir la distribución de la información y la comunicación en toda la organización. Esta 
estrategia innovadora es la que soporta, por tanto, los resultados de los proyectos de innovación que tienen impacto tanto en la empresa como en la obra.

Los resultados obtenidos en la investigación de una empresa constructora, así como con las observaciones y datos obtenidos de otras empresas y profesionales del sector permitieron formular las 18 proposiciones que se exponen en la Tabla 1 (Pellicer et al. 2012). Estas proposiciones se organizaron de acuerdo con aspectos clave identificados en el proceso de gestión de la innovación (Correa et al. 2007, Pellicer et al. 2012): inductores de la innovación, resultados de la innovación, sistema de innovación, ambiente empresarial y capacidades organizativas (véase la Figura 1). La Tabla 1 también incluye las referencias bibliográficas básicas que apoyan la formulación de cada una de las proposiciones del estudio de caso. Esta investigación cualitativa se llevó a cabo siguiendo el proceso propuesto por Yin (2003). La empresa seleccionada se denominó Lambda, a efectos de salvaguardar su identidad.

$<$ TABLA $1>$

Un sistema de gestión de la innovación transforma inductores de innovación en resultados y beneficios concretos. El sistema viene influenciado por el ambiente empresarial y las capacidades organizativas de la empresa. La gestión de la innovación comienza con la identificación de oportunidades provenientes tanto de los requerimientos de los agentes participantes (empleados, clientes, suministradores y el entorno), como de las dificultades que pueden aparecer en la obra. Las mejores ideas se seleccionan por la dirección de la empresa para transformarse en proyectos de innovación. El departamento responsable de la innovación organiza y asigna los recursos necesarios, implementa y supervisa el conjunto de proyectos. 
Estas innovaciones se evalúan y codifican, pasando a ser lecciones aprendidas que deben transferirse a futuros proyectos. Los resultados de la innovación se aplican en las obras o en la propia empresa; estos resultados proporcionan una retroalimentación fundamental para el ciclo de mejora continua. Este proceso de gestión de la innovación se describe con detalle en Yepes et al. (2010).

\section{VALIDACIÓN DE LAS PROPOSICIONES}

Tal y como se comenta en la Introducción, el objetivo de esta investigación es validar el modelo explicativo, y basado en evidencias, de la gestión de la innovación. Este modelo teórico se desarrolló analizando una empresa constructora española durante un período de cinco años mediante el método de investigación del estudio de caso (Yin 2003). La estrategia de estudio de caso único fue la elegida porque la investigación arrancó al tiempo que el gobierno español emitió la norma UNE 166002 (AENOR 2006, Pellicer et al., 2008, 2010). Una descripción exhaustiva de la empresa se encuentra en Pellicer et al. (2010), mientras que la elección de esta empresa como estudio de caso único se justifica plenamente en Pellicer et al. (2012).

El proceso de investigación llevado a cabo en el estudio de caso sigue la propuesta de Yin (2003). Este proceso incluye seis pasos: (a) revisión de la literatura, (b) diseño de un modelo lógico, (c) recopilación de datos, (d) análisis de datos, (e) exposición de resultados, y (f) validación de resultados. Los primeros cinco pasos comparan sistemáticamente la teoría con la realidad desde diferentes puntos de vista, utilizando múltiples fuentes de evidencia, detallando los hechos percibidos de un modo lógico, y construyendo explicaciones de los 
hechos analizados. Para cumplir con este objetivo, se utilizaron diferentes técnicas (Pellicer et al. 2012): (a) observación participante; (b) reuniones mensuales con directivos de la empresa; (c) análisis de documentos internos elaborados en la empresa; (d) cuatro encuestas a empleados de la empresa; (e) una encuesta a sus suministradores y subcontratistas; y (f) seminarios anuales con expertos y gestores relevantes.

Con el fin de garantizar la calidad de la investigación, Yin (2003) propone el cumplimiento de cuatro pruebas: (1) validación de las construcciones conceptuales, (2) validación interna, (3) validación externa, y (4) fiabilidad de los resultados. A continuación se explica su aplicación a esta investigación. La validez de las construcciones conceptuales supone la utilización de múltiples fuentes de información y el encadenamiento de evidencias; ambas se aplicaron durante la etapa de recopilación de datos. La validez interna se refiere a la lógica de la causalidad del estudio cualitativo; según Yin (2003), ésta se consigue de dos modos: construyendo explicaciones del fenómeno en estudio (“explanation-building”) y contrastando lo que predice la teoría con la realidad observada (“pattern-matching”). La validez externa es el principal objetivo de la investigación reflejada en este artículo. Finalmente, la fiabilidad se consigue mediante un protocolo previo del caso y la existencia de una base de datos con todo el material y evidencias obtenidas.

Tal y como se ha expuesto, el proceso investigador de un estudio de caso exige una validación externa (Yin 2003); este es el objetivo de este artículo. Esto significa la comprobación de las proposiciones expuestas anteriormente para que el modelo se pueda generalizar al universo de empresas constructoras con un sistema implantado de gestión de la innovación. Para ello, se realizaron entrevistas externas a directivos de siete empresas constructoras españolas que tuviesen su sistema de gestión de la innovación certificado según 
la norma UNE 166002 (véase la Tabla 2). La población de empresas certificadas hasta ese momento era de ocho, por lo que se consideró que la muestra era representativa. Los entrevistados eran directores del departamento responsable de la innovación en la empresa (además también responsables de la gestión de la calidad en cuatro de las empresas), con una experiencia mínima de 15 años en el sector de la construcción y con titulaciones universitarias de ingeniero civil (cuatro casos) o ingeniero industrial (tres casos). Estas entrevistas se estructuraron en tres etapas:

1. Obtención de los datos básicos que caracterizan a estas empresas (resumidos en la Tabla 2).

2. Validación de las proposiciones mediante respuestas a un cuestionario (incluido en el Apéndice).

3. Profundización del conocimiento sobre las barreras y beneficios del proceso de innovación mediante una entrevista dirigida de una duración mínima de 120 minutos por empresa.

$<$ TABLA 2>

Además, se realizaron encuestas a ocho directivos de la empresa Lambda y a nueve expertos, independientes de todas estas empresas, del sector de la construcción. Los directivos de la empresa Lambda eran todos directores de departamento con 10 años de experiencia mínima en el sector de la construcción; siete de ellos eran ingenieros civiles y uno de ellos ingeniero químico. Los expertos incluyen representantes de diferentes organizaciones: suministrador de materiales, consultor independiente, empresa consultora, promotor privado, administración central, administración local, asociación profesional, organismo de certificación y profesor universitario; todos ellos tenían una experiencia mínima de 20 años trabajando en el sector. 
Siete de ellos eran ingenieros civiles y dos arquitectos. La entrevista fue contrastada y refinada previamente mediante una prueba piloto realizada a tres profesores universitarios con más de 20 años de experiencia profesional en el sector de la construcción.

El grado de aceptación de las proposiciones se obtuvo del análisis de las respuestas por grupos encuestados: empresas certificadas (7), directivos de Lambda (8) y expertos (9). En el Apéndice se incluye el cuestionario completo; la proposición relacionada con cada pregunta se indica entre paréntesis con la finalidad de facilitar el análisis del cuestionario.

Las alternativas de respuesta se escalaron de modo que pudiera calcularse el promedio de cada grupo. Aquellas preguntas con alternativas de respuesta "elevado”, "intermedio" y “reducido" recibieron valores 2, 1 y 0, respectivamente. En cambio, a las respuestas con alternativas “muy de acuerdo”, “de acuerdo”, en “desacuerdo” y “muy en desacuerdo” se le asignaron valores 2, 1, -1 y -2, respectivamente. Tomando estas referencias, se calculó el promedio para cada pregunta y grupo. Se categorizó de este modo una proposición como “fuerte" (F) cuando la media obtenida fue superior a 1.3 y "débil” (D) cuando le media fue inferior a 0.7. Para situaciones intermedias, la proposición se categorizó con un grado de validación “medio” (M).

\section{RESULTADOS}

Todas las proposiciones recibieron un grado de respaldo “fuerte”, para todos los grupos encuestados, excepto las proposiciones reflejadas en la Tabla 3. En esta tabla se indica la proposición afectada (por su código), y el grado de conformidad obtenido para cada grupo 
entrevistado: fuerte, medio o débil. Puede observarse en la Tabla 3 que los resultados obtenidos para las empresas y para los directivos de Lambda concuerdan plenamente. Estos resultados se representan en una gráfica (Figura 1) del siguiente modo: (a) fuerte (letra negrita y trazo muy resaltado); (b) medio (letra y trazo normales); y (c) débil (letra cursiva y trazo fino). Esta coincidencia señala la alineación existente entre la realidad de la empresa y sus directivos en relación con la innovación. Sin embargo, existen discrepancias con los expertos, puesto que éstos valoran cuatro de las proposiciones con un nivel de conformidad inferior, mientras que sólo la proposición $\mathrm{P}_{4}$ una obtiene una mayor aceptación (medio). Es de destacar que precisamente esta proposición fue la única que obtuvo un asentimiento débil por parte de las empresas certificadas.

$<$ TABLA 3>

$<$ FIGURA 1 $>$

De las entrevistas realizadas pueden extraerse otros resultados interesantes, no relacionados directamente con la validación del modelo, que se indican a continuación:

- Cuatro empresas señalan que los organismos certificadores asocian la innovación con la investigación científica. Esta indefinición en el alcance de la norma dificulta la justificación de determinadas innovaciones tecnológicas.

- Un aspecto señalado reiteradamente por las empresas es el conflicto generado cuando las necesidades de los clientes se alejan de la normativa o de las prácticas habituales en la construcción.

- Existen evidencias que relacionan la innovación, la calidad y la gestión del conocimiento. Dos de las empresas desplazan equipos de especialistas para implantar "in situ” los 
resultados de la innovación. Si ésta funciona, elaboran nuevos procedimientos de trabajo que se añaden al sistema de calidad, retroalimentando el conocimiento generado.

- Las empresas destacan la necesidad e importancia de la vigilancia tecnológica en la generación de ideas innovadoras, a pesar de la dificultad.

\section{DISCUSIÓN}

El análisis de los resultados muestra que, en general, el grado de validación del modelo planteado es muy alto considerando que 15 de las 18 proposiciones fueron fuertemente valoradas, dos de ellas tuvieron un nivel de conformidad medio y sólo una lo tuvo débil. La Figura 1 resume gráficamente los resultados; éstos fueron avalados por los directivos de las empresas externas y los de la empresa estudiada. La discusión en esta sección se centra principalmente en analizar las proposiciones cuya corroboración fue media y débil, analizando aquellos casos donde se detectaron ligeras discrepancias en las respuestas de los expertos externos respecto a los directivos de las empresas constructoras.

Existe un consenso generalizado en cuanto a la influencia de los inductores de la innovación. No obstante, la influencia de los resultados de salida del proceso tanto en la empresa constructora como en sus proyectos no resulta tan evidente; la única excepción es el aumento de las capacidades tecnológicas expuestas en la proposición $\mathrm{P}_{14}$. La proposición $\mathrm{P}_{4}$ plantea que "la adopción de un sistema de gestión de la innovación en una empresa constructora permite innovar siguiendo una estrategia previamente definida”; muestra una aceptación débil, a pesar de que su importancia se ha destacado en estudios previos (Nam y Tatum 1992, Eaton 2001, Seaden et al. 2003, Sexton y Barrett 2003, Taylor y Levitt 2004, Hartmann 2006, 
Lim et al. 2010). Resulta difícil renunciar a una explicación causa-efecto entre la adopción de estrategias enfocadas a la innovación a través de un sistema de gestión y la consecución de resultados innovadores. Las empresas no reconocen la existencia de una estrategia previa y específica de innovación. No obstante, esto no ha sido un obstáculo para las empresas que han certificado su sistema de gestión de la innovación. Así pues, existen estrategias empresariales de innovación informales en etapas no maduras de gestión de los procesos de innovación.

Un segundo nivel de discrepancia viene reflejado en las proposiciones $\mathrm{P}_{15}$ y $\mathrm{P}_{16}$. Tanto las empresas encuestadas, como los directivos de Lambda o los expertos consultados no perciben con nitidez la relación entre la adopción de un sistema de gestión de la innovación y el incremento de la competitividad de la empresa constructora $\left(\mathrm{P}_{15}\right)$. Esta percepción también es evidente en otros ámbitos de la gestión empresarial donde, la simple certificación de un sistema de gestión de la calidad a través de la norma ISO 9001 tampoco garantiza un incremento de la competitividad en una empresa. Por tanto, si bien la adopción de un sistema de gestión de la innovación ayuda a mejorar la competitividad de una empresa constructora, esta medida parece por sí sola insuficiente para conseguir dicho objetivo. En segundo lugar, tampoco se ha confirmado de forma rotunda la proposición $\mathrm{P}_{16}$ que afirma que "la certificación de un proyecto de innovación mejora los resultados de una obra”. En este caso la encuesta a los expertos ha dado como resultado una relación débil frente a la relación media del resto de grupos. Una posible interpretación deriva de los resultados económicos exigidos a corto plazo en las obras. La innovación genera beneficios que no son sólo económicos; este beneficio se puede expandir a toda la organización con un adecuado sistema de gestión del conocimiento. Existen ventajas fiscales, organizativas y competitivas que a veces es difícil plasmar en puros beneficios económicos para una obra determinada. Por tanto, si bien parece 
evidente que la innovación contribuye a la obtención de resultados en una empresa constructora, y por ende, en sus obras, el corto plazo puede distorsionar la visibilidad de la relación causa-efecto entre innovación y resultados económicos. Esta distorsión se acentúa cuando el éxito de una obra queda sujeto, en numerosas ocasiones, a factores ajenos a la propia innovación.

En un tercer nivel de discrepancias, sólo los expertos han diferido levemente respecto a la visión interna del proceso de gestión de la innovación. En este sentido, la proposición $\mathrm{P}_{2}$ afirma que "la empresa constructora innova para cumplir con los requerimientos de los clientes”. Esto puede ser debido a la relación cercana que mantienen las empresas constructoras con sus clientes; esta relación no la tienen los expertos. Si bien existen otras fuentes de innovación, parece evidente que son los requerimientos de los clientes una de las razones de mayor peso para hacerlo. Esta pequeña discrepancia entre los expertos y el resto de grupos también se manifiesta en la proposición $\mathrm{P}_{7}$. Esta afirma que "la implantación de un sistema de gestión de la innovación mejora la gestión del conocimiento”. Para los expertos la conformidad con esta proposición no es fuerte, quizá porque no disponen de la experiencia de las empresas certificadas, donde el simple hecho de normalizar la innovación ha permitido abrir mecanismos de comunicación horizontal y vertical en la empresa que han facilitado enormemente el flujo de información y de conocimiento.

Por todo lo anteriormente expuesto, la validación suficientemente elevada de las proposiciones que conforman el modelo propuesto permite clarificar la forma y los motivos que impulsan la gestión de la innovación en las empresas constructoras. Las discrepancias existentes, fundamentalmente expresadas en las proposiciones $\mathrm{P}_{4}, \mathrm{P}_{15}$ y $\mathrm{P}_{16}$, pueden explicarse como consecuencia de la poca experiencia acumulada en la gestión de los procesos 
de innovación de estas empresas dentro de esquemas de gestión normalizados. Además, la escasa visibilidad de las ventajas competitivas a largo plazo también queda oscurecida dentro de la organización cuando los procesos de innovación están basados en estrategias informales.

Finalmente, respecto a las barreras a la innovación, la relativa a la priorización de los procesos productivos por parte de la empresa $\left(\mathrm{P}_{18}\right)$ es completamente respaldada por todos los grupos. Es percibida como la principal barrera para innovar en la construcción, y ha sido corroborada por todos los entrevistados. La otra barrera identificada es la infravaloración de la innovación como estrategia competitiva por parte de los líderes de la empresa $\left(\mathrm{P}_{17}\right)$. Esta proposición presenta discrepancias entre los grupos porque los expertos tampoco han considerado decisiva la influencia del personal directivo en la innovación. Esta visión de que la estrategia competitiva basada en la innovación debe pasar por el compromiso de la alta dirección es un hecho claramente contrastado en las empresas certificadas. Esto puede quedar algo oscurecido en opinión de los expertos, más proclives a acentuar la influencia del personal técnico en los aspectos relacionados con la innovación.

\section{CONCLUSIONES, LIMITACIONES Y FUTURAS INVESTIGACIONES}

Este artículo presenta el proceso de validación de un modelo de gestión de la innovación para empresas constructoras; está basado en la investigación desarrollada mediante el estudio de caso de una empresa constructora de tamaño medio, así como la cooperación con otras empresas y profesionales del sector de la construcción español. Para ello, se sometieron 18 proposiciones al juicio de directivos de empresas certificadas en gestión de la innovación, 
directivos de la empresa estudiada y expertos independientes. El resultado de esta validación fue un amplio grado de consenso entre los diferentes grupos de entrevistados y una aceptación fuerte de 15 de las proposiciones planteadas. Como consecuencia de la investigación realizada, se puede concluir lo siguiente:

- Los problemas técnicos en las obras, los requerimientos de los clientes y la alta dirección, son las principales fuentes de innovación en las empresas constructoras.

- Las constructoras innovan fundamentalmente en procesos y en sus productos relacionados.

- La detección de las oportunidades de innovación es el resultado de la vigilancia tanto de los procesos internos de la empresa, como de la obra y del entorno.

- Identificar, desarrollar y transferir una solución innovadora requiere integrar varias disciplinas:

$\checkmark$ La observación del entorno, incluida la vigilancia tecnológica, para buscar oportunidades de innovación, soluciones factibles y socios tecnológicos que añadan valor al proceso de innovación.

$\checkmark$ La gestión del conocimiento de la organización puede transferir lo hallado a otros proyectos, tanto constructivos como de innovación.

$\checkmark$ La habilidad para detectar los requerimientos de los clientes.

- La colaboración con socios tecnológicos y la gestión de equipos multidisciplinares son condiciones necesarias para innovar en las empresas constructoras.

- La implantación de un sistema de gestión de la innovación se beneficia de la existencia previa de un sistema de gestión de la calidad.

- El incremento de la capacidad técnica es el principal beneficio de la gestión de la innovación. 
- Las dos barreras principales para innovar son las priorización de los procesos productivos y la infravaloración de la innovación como estrategia competitiva por la alta dirección

La proposición menos respaldada indica que la adopción de un sistema de gestión de la innovación permite innovar siguiendo una estrategia previamente definida; esto puede suceder debido a la existencia de estrategias de innovación informales en etapas no maduras de gestión de la innovación. Tampoco se percibe con transparencia la relación entre la adopción de un sistema de gestión de la innovación y el incremento de la competitividad de la empresa constructora. Esta situación puede estar asociada a las etapas tempranas en que se encontraban estos procesos cuando se desarrolló la investigación. Por otra parte, los expertos no aprecian, con la nitidez de las empresas, la importancia de las demandas de los clientes, la influencia del personal directivo en el proceso de innovación, o el impacto positivo de la innovación en la gestión del conocimiento de las empresas.

Este modelo puede ayudar a los profesionales (a nivel de proyecto o de empresa) a sistematizar y homogeneizar sus procesos de innovación, si bien la norma UNE 166002 (u otra similar) no es necesaria específicamente para este propósito. Un enfoque de gestión sistemático permite planificar, organizar, dirigir y controlar la innovación como cualquier otra actividad empresarial en una empresa constructora. Este enfoque facilita la asimilación de nuevo conocimiento e ideas. Por lo tanto, su implantación en las empresas constructoras puede mejorar tanto la resolución de problemas en la organización de un modo metódico como las capacidades técnicas y tecnológicas de los empleados, en particular, y de la empresa, en general. 
Las conclusiones de esta investigación se encuentran limitadas por la escasa experiencia acumulada en la normalización de estos sistemas. Por lo tanto, el modelo propuesto debería ser comprobado o mejorado en el futuro con un mayor número de empresas, más maduras en la gestión de la innovación, y que dispongan de sistemas certificados externamente. La influencia de la variable temporal en los resultados obtenidos y los condicionantes locales son aspectos que deben analizarse con mayor profundidad en futuras investigaciones, ya en curso. Por una parte, se están estudiando varios casos de empresas constructoras chilenas para contrastar el grado de madurez de la gestión de la innovación y el entorno empresarial en un ámbito distinto al analizado. Por otro lado, se está llevando a cabo una encuesta de mayor alcance a las empresas constructoras españolas que ya tienen certificados sus sistemas de gestión de la innovación.

\section{AGRADECIMIENTOS}

Esta investigación fue parcialmente financiada por la Universidad Católica del Maule (proyecto MECESUP-UCM0205), el Ministerio de Fomento del Gobierno de España (proyecto 2004-36) y la Universitat Politècnica de València (contrato UPV-20050921). Los autores agradecen a Ricardo Lacort, Francisco J. Vea y Manuel Civera toda la colaboración y el apoyo prestado, y a José C. Teixeira las sugerencias aportadas. Asimismo, los autores agradecen a los revisores anónimos sus sugerencias y comentarios constructivos. 


\section{APÉNDICE: CUESTIONARIO}

1. Las empresas constructoras pueden desarrollar proyectos de innovación con el propósito de (indicar si “elevado”, “intermedio” o “reducido”): (a) Acceder a nuevos mercados u obtener una mayor cuota de mercado; (b) Resolver problemas técnicos de la obra ( $\left.\mathrm{P}_{1}\right)$; (c) Responder a los requerimientos de los clientes $\left(\mathrm{P}_{2}\right)$; (d) Incrementar la calidad de sus infraestructuras; (e) Mejorar la competitividad de la empresa ( $\left.\mathrm{P}_{3}\right)$; (f) Otra.

2. ¿Ha realizado su empresa alguno de los siguientes tipos de innovación? (indicar si en grado “elevado”, “intermedio” o “reducido”) ( $\left.\mathrm{P}_{6}\right)$ : (a) Producto; (b) Procesos; (c) Organizativa; (d) Mercadotecnia.

3. Los siguientes enunciados hacen referencia a aspectos de un sistema de gestión de la innovación (indicar su grado de conformidad si “muy de acuerdo”, “de acuerdo”, “en desacuerdo” o “muy en desacuerdo”): (a) La implantación de un sistema de gestión de la innovación mejora la gestión del conocimiento de una empresa constructora $\left(\mathrm{P}_{7}\right)$; (b) Las organizaciones que adoptan un sistema de gestión de la innovación conocen mejor su entorno externo $\left(\mathrm{P}_{8}\right)$; (c) La existencia de un sistema de calidad certificado según la norma UNE 9001 facilita la implantación de un sistema de gestión de la innovación $\left(\mathrm{P}_{10}\right)$; (d) La innovación necesita de la participación de equipos multidisciplinares ( $\left.\mathrm{P}_{13}\right)$; (e) La implicación activa del jefe de obra en el proceso innovador tiene un impacto significativo en los resultados de la innovación ( $\left.\mathrm{P}_{12}\right)$; (f) La certificación de un proyecto de innovación mejora los resultados de la obra $\left(\mathrm{P}_{16}\right)$; (g) El control de los procesos internos (producción, gestión, etc.) es fundamental para innovar $\left(\mathrm{P}_{9}\right)$; (h) La existencia de un sistema de gestión de la innovación facilita la subcontratación de empresas especializadas que aporten valor 
al proceso innovador ( $\left.\mathrm{P}_{11}\right)$; (i) La implantación de un sistema de innovación en la empresa constructora obedece a la necesidad de generar una diferenciación positiva que valoren los clientes $\left(\mathrm{P}_{5}\right)$; (j) La adopción de un sistema de gestión de la innovación incrementa la competitividad de la empresa constructora $\left(\mathrm{P}_{15}\right)$; $(\mathrm{k})$ La adopción de un sistema de gestión de la innovación mejora la capacidad técnica de la empresa constructora $\left(\mathrm{P}_{14}\right)$; (l) Para que una empresa constructora innove siguiendo una estrategia predefinida se requiere un sistema de gestión de la innovación $\left(\mathrm{P}_{4}\right)$.

4. ¿Cuáles son las principales barreras para innovar? (indicar si en grado "elevado", “intermedio” o “reducido”): (a) Priorización de los procesos productivos $\left(\mathrm{P}_{18}\right)$; (b) Falta de incentivos; (c) Carencia de una cultura adecuada; (d) Infravaloración de la innovación como estrategia competitiva $\left(\mathrm{P}_{17}\right)$; (e) Falta de liderazgo en innovación $\left(\mathrm{P}_{17}\right)$; (f) Escasez de personal capacitado en innovación; (g) Otros.

\section{REFERENCIAS}

AENOR (2006) UNE 166002:2006. R\&D\&i management: requirements for $R \& D \& i$ management systems. AENOR, Madrid.

Blayse, A.M., Manley, K. (2004) “Key influences on construction innovation”. Construction Innovation, 4, 143-154.

Bossink, B.A.G. (2004) “Managing drivers of innovation in construction networks”. Journal of Construction Engineering and Management, 130(3), 337-345

Casadesus, M., Karapetrovic, S., Heras, I. (2011) “Synergies in standardized management systems: some empirical evidence”. The TQM Journal, 23(1), 73-86. 
Coelho, D.A., Matias, J.C.O. (2010) “An empirical study on integration of the innovation management systems (MS) with other MSs within organizations”. Proceedings of ERIMA, pp. 5-13.

Correa, C.L., Pellicer, E., Yepes, V. (2007) “Factores determinantes y propuestas para la gestión de la innovación en las empresas constructoras”. Revista Ingeniería de Construcción, 22(1), 5-14.

Davenport, T.H. (2005) “The coming commoditization of processes". Harvard Business Review, 83(6), 101-108.

Dikmen, I., Birgonul, M.T., Artuk, S.U. (2005) "Integrated framework to investigate value innovations”. Journal of Management in Engineering, 21(2), 81-89.

Dulaimi, M. (1995) “The challenge of innovation in construction”. Building Research and Information, 23(2), 106-109.

Eaton, D. (2001) “A temporal typology for innovation within the construction industry”. Construction Innovation, 1(3), 165-179.

Evangelista, R., Perani, G., Rapiti, F., Archibugi, D. (1997) “Nature and impact of innovation in manufacturing industry: some evidence from the Italian innovation survey”. Research Policy, 26 (4-5), 521-536.

Gann, D.M. (1997) “Should governments fund construction research?”. Building Research \& Information, 25, 257- 267.

Gann, D.M. (2000) Building innovation. Complex constructs in a changing world. Thomas Telford Publishing, London.

Gann, D.M., Salter A. (2000) "Innovation in project-based, service-enhanced firms: the construction of complex products and systems”. Research Policy, 29 (7-8), 955-972. 
Gibbons, M., Limoges, C., Nowotny, H., Schwartzman, S. (1994) The new production of knowledge: the dynamics of science and research in contemporary societies. Sage Publications, London.

Hardie, M.P., Miller, G., Manley, K.J., McFallan, S. (2005) "Experience with the management of technological innovations within the Australian construction industry”. Proceedings of the International Conference on Management of Engineering and Technology, 244-250.

Hartmann, A. (2006) “The context of innovation management in construction firms". Construction Management and Economics, 24(6), 567-578.

Harty, C. (2008) "Implementing innovation in construction: contexts, relative boundedness and actor-network theory”. Construction Management and Economics, 26, 10291041.

Kangari, R., Miyatake, Y. (1997) "Developing and managing innovative construction technologies in Japan”. Journal of Construction Engineering and Management, 123(1), 72-78.

Ko, R.K.L. (2009) “A computer scientist's introductory guide to business process management (BPM)”. Crossroads, 15(4), 11-18.

Kornish, L.J., Ulrich, K.T. (2011) “Opportunity spaces in innovation: Empirical analysis of large sample of ideas”. Management Science, 57(1), 107-128.

Lim, J.N., Schultmann, F., Ofori, G. (2010) “Tailoring competitive advantages derived from innovation to the needs of construction firms”. Journal of Construction Engineering and Management, 136(5), 568-580.

Manseau, A. (1998) “Who cares about overall industry innovativeness?”. Building Research and Information, 26(4), 241-245. 
Marimon, F., Cristobal, E. (2005) “A study on ISO 9000 certification process: Consultant profiles and company behaviour”. Managing Service Quality, 15(3), 290-305.

Mir, M., Casadesus, M. (2011) “Normas para la gestión de la innovación: Un análisis comparativo”. DYNA, 86(1), 49-58.

Mitropoulos, P., Tatum, C.B. (2000) "Forces driving adaptation of new information technologies”. Journal of Construction Engineering and Management, 126(5), 340348.

Nam, C.H., Tatum, C.B. (1992) "Strategies for technology push: Lessons from construction innovations”. Journal of Construction Engineering and Management, 118, 507-524.

Nam, C.H., Tatum, C.B. (1997) “Leaders and champions for construction innovation”. Construction Management and Economics, 15(3), 259-270.

Parikh, M. (2001) "Knowledge management framework for high-tech research and development”. Engineering Management Journal, 13(3), 27-33.

Park, M.S., Nepal, M.P., Dulaimi, M.F. (2004) “Dynamic modelling for construction innovation”. Journal of Management in Engineering, 20(4), 171-177.

Pellicer, E., Correa, C.L., Yepes, V., Alarcón, L.F. (2012) “Organizational improvement through standardization of the innovation process in construction firms”. Engineering Management Journal, 24(2), 40-53.

Pellicer, E., Yepes, V., Correa, C.L., Martinez, G. (2008) "Enhancing R\&D\&i through standardization and certification: The case of the Spanish construction industry”. Revista Ingeniería de Construcción, 23(2), 112-119.

Pellicer, E., Yepes, V., Rojas, R.J. (2010) “Innovation and competitiveness in construction companies: A case study”. Journal of Management Research, 10(2), 103-115.

Perdomo-Ortiz, J., Gonzalez-Benito, J., Galende, J. (2006) “Total quality management as a forerunner of business innovation capability”. Technovation, 26, 1170-1185. 
Perdomo-Ortiz, J., Gonzalez-Benito, J., Galende, J. (2009) “The intervening effect of business innovation capability on the relationship between total quality management and technological innovation”. International Journal of Production Research, 47(18), 5087-5107.

Prajogo, D.I., Sohal, A.S. (2006) “The integration of TQM and technology/R\&D management in determining quality and innovation performance”. Omega, 34(3), 296312.

Pries, F., Janszen, F. (1995) "Innovation in the construction industry: The dominant role of the environment”. Construction Management and Economics, 13(1), 43-51.

Santos-Vijande, M.L., Alvarez-Gonzalez, L.I. (2007) “Innovativeness and organizational innovation in total quality oriented firms: The moderating role of market turbulence”. Technovation, 27(9), 514-532.

Seaden, G., Guolla, M., Doutriaux, J., Nash, J. (2003) "Strategic decisions and innovation in construction firms”. Construction Management and Economics, 21(6), 603-612.

Seaden, G., Manseau, A. (2001) "Public policy and construction innovation”. Building Research and Information, 29(3), 182-196.

Serpell, A. (2001) "Innovation in the Chilean construction industry: Public policy instruments”. In: Innovation in Construction: An international Review of Public Policy (Eds. A. Manseau and G. Seaden), Spon Press, London, 111-128.

Sexton, M., Barrett, P. (2003) “A literature synthesis of innovation in small construction firms: insights, ambiguities and questions”. Construction Management and Economics, 21(6), 613-622.

Slaughter, S. (1993) “Innovation and learning during implementation: A comparison of user and manufacturer innovations”. Research Policy, 22 (1), 81-95. 
Slaughter, S. (2000) “Implementation of construction innovations”. Building Research and Information, 28(1), 2-17.

Stewart, I., Fenn, P. (2006) "Strategy: the motivation for innovation". Construction Innovation, 6, 173-185.

Tatum, C.B. (1987) "Process of innovation in construction firms". Journal of Construction Engineering and Management, 113(4), 648-663.

Taylor, J.E., Levitt, R.E. (2004) "Understanding and managing innovations in project-based industries”. Innovations - Project Management Research 2004. Project Management Institute, New York.

Teixeira, J.C, Pellicer, E., Pedro, P., Yepes, V. (2009) “Fostering research, development and innovation in construction companies”. CIB Joint International Symposium 2009 Construction Facing Worldwide Challenges, Dubrovnik, 27 September-1 October.

Tidd, J., Bessant, J., Pavitt, K. (1997) Managing innovation: integrating technological, market and organizational change. John Wiley \& Sons, Chichester, UK.

Trkman, P. (2010) “The critical success factors of business process management”. International Journal of Information Management, 30, pp.125-134.

Van de Ven, A.H., Poole, M.S. (2005) “Alternative approaches for studying organizational change”. Organization Studies, 26(9), 1377-1404.

Vanhaverbeke, W., Torremans, H. (1999) “Organizational structure in process-based organizations”. Knowledge and Process Management, 6(1), 41-52.

Vea, F.J., Pérez, J., Pellicer, E., Yepes, V. (2010) "Sistema de control dimensional y de replanteo de alta precisión de elementos prefabricados singulares”. Revista de la Construcción, 9(2), 116-125.

Veugelers, R., Cassiman, B. (1999) “Make and buy in innovation strategies: evidence from Belgian manufacturing firms”. Research Policy, 28 (1), 63-80. 
Wagner, S.M. (2003) “Intensity and managerial scope of supplier integration”. Journal of Supply Chain Management, 39(4), 4-15.

Winch, G.M. (1998) "Zephyrs of creative destruction: understanding the management of innovation in construction”. Building Research and Information, 26(5), 268-279.

Yepes, V., Pellicer, E., Correa, C.L., Alarcon, L.F. (2010) "Implementing a system for achieving innovation opportunities in a construction company”. Proceedings of the CIB World Congress 2010 - Building a Better World, 319-330.

Yin, R. (2003) Case study research: Design and methods, $3^{\text {rd }}$ Ed. Sage Publications, London. 


\section{ENTORNO DE NEGOCIOS}

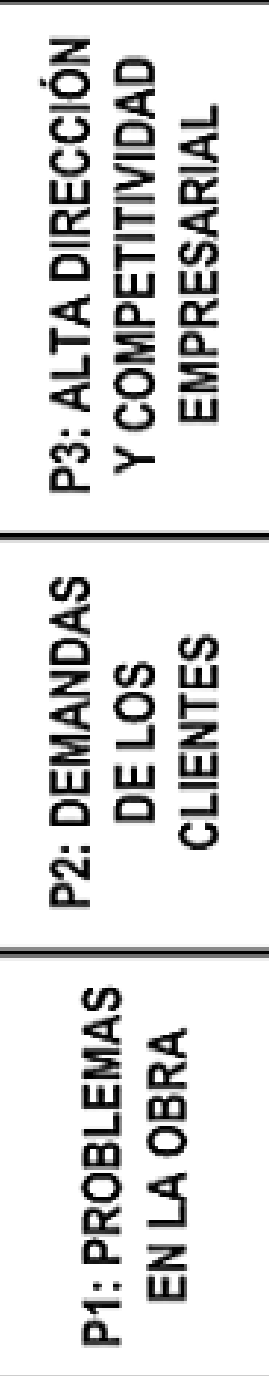
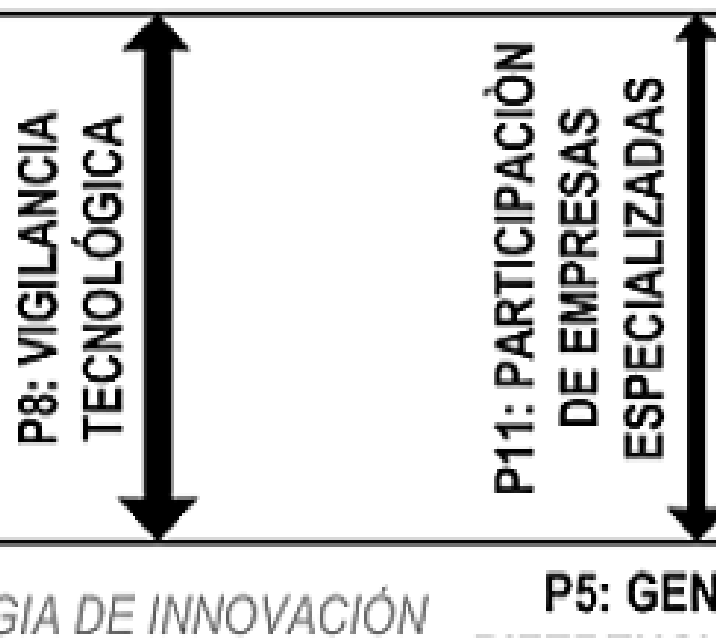

P4: ESTRATEGIA DE INNOVACIÓN

P5: GENERACIÓN DE

P6: INNOVACIÓN EN PROCESOS DIFERENCIACIÓN POSITIVA P9: CONTROL DE LOS PROCESOS INTERNOS

P17: PERCEPCIÓN DE LA INNOVACIÓN P18: PRIORIZACIÓN DE LOS COMO ESTRATEGIA COMPETITIVA PROCESOS PRODUCTIVOS SISTEMA DE INNOVACIÓN
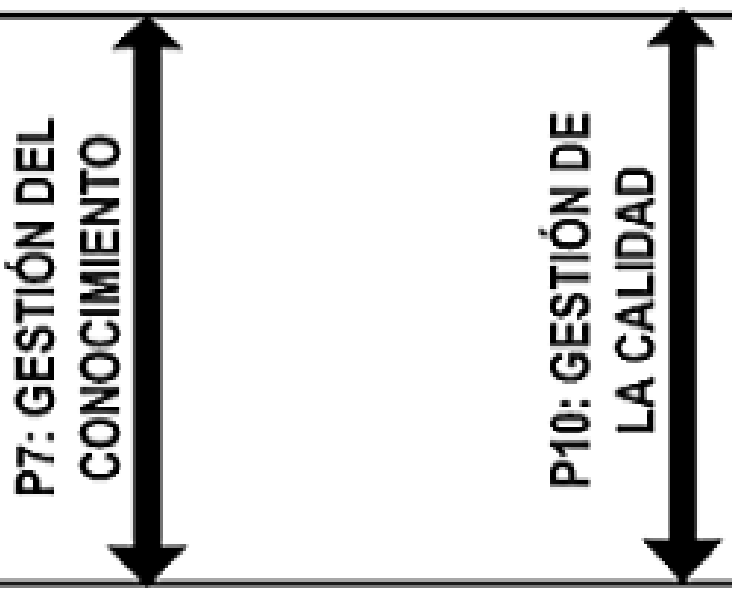

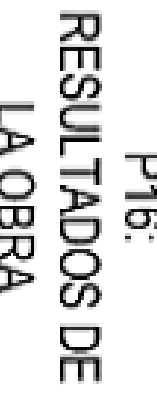

\section{CAPACIDADES ORGANIZATIVAS}

P12: JEFE DE OBRA

P13: EQUIPOS MULTIDISCIPLINARES 


\begin{tabular}{|c|c|c|}
\hline CÓDIGO & PROPOSICIÓN & REFERENCIAS \\
\hline $\mathbf{P}_{1}$ & $\begin{array}{l}\text { La principal fuente de innovación para la empresa } \\
\text { constructora proviene de los problemas técnicos que } \\
\text { aparecen en la ejecución de la obra. }\end{array}$ & $\begin{array}{l}\text { Slaughter (1993), Nam y Tatum (1997), } \\
\text { Winch (1998) }\end{array}$ \\
\hline $\mathbf{P}_{2}$ & $\begin{array}{l}\text { La empresa constructora innova para cumplir con los } \\
\text { requerimientos de los clientes. }\end{array}$ & $\begin{array}{l}\text { Nam y Tatum (1997), Mitropoulus y } \\
\text { Tatum (2000), Blayse y Manley (2004) }\end{array}$ \\
\hline $\mathbf{P}_{3}$ & $\begin{array}{l}\text { La dirección impulsa proyectos de innovación para } \\
\text { mejorar la competitividad de la empresa constructora. }\end{array}$ & $\begin{array}{l}\text { Tatum (1987), Winch (1998), Slaughter } \\
\text { (2000) }\end{array}$ \\
\hline $\mathbf{P}_{4}$ & $\begin{array}{l}\text { La adopción de un sistema de gestión de la innovación } \\
\text { en la empresa constructora permite innovar siguiendo } \\
\text { una estrategia previamente definida. }\end{array}$ & $\begin{array}{l}\text { Gann y Salter (2000), Seaden et al. } \\
\text { (2003), Stewart y Fenn (2006) }\end{array}$ \\
\hline $\mathbf{P}_{5}$ & $\begin{array}{l}\text { La implantación de un sistema de innovación en la } \\
\text { empresa constructora obedece a la necesidad de generar } \\
\text { una diferenciación positiva que valoren los clientes. }\end{array}$ & $\begin{array}{l}\text { Slaughter (2000), Sexton y Barrett } \\
\text { (2003), Van den Ven y Poole (2005) }\end{array}$ \\
\hline $\mathbf{P}_{6}$ & $\begin{array}{l}\text { La empresa constructora innova fundamentalmente en } \\
\text { los procesos. }\end{array}$ & $\begin{array}{l}\text { Gann y Salter (2000), Sexton y Barrett } \\
\text { (2003) }\end{array}$ \\
\hline $\mathbf{P}_{7}$ & $\begin{array}{l}\text { La implantación de un sistema de gestión de la } \\
\text { innovación mejora la gestión del conocimiento. }\end{array}$ & $\begin{array}{l}\text { Winch (1998), Parikh (2001), Hardie et } \\
\text { al. (2005) }\end{array}$ \\
\hline $\mathbf{P}_{\mathbf{8}}$ & $\begin{array}{l}\text { La empresa constructora que adopta un sistema de } \\
\text { gestión de la innovación conoce mejor su entorno } \\
\text { externo. }\end{array}$ & $\begin{array}{l}\text { Tatum (1987), Pries y Janszen (1995), } \\
\text { Seaden et al. (2003) }\end{array}$ \\
\hline $\mathbf{P}_{9}$ & $\begin{array}{l}\text { El control de los procesos internos de la empresa } \\
\text { (producción y gestión fundamentalmente) constituye } \\
\text { una fuente de información básica para la generación de } \\
\text { ideas innovadoras. }\end{array}$ & $\begin{array}{l}\text { Dulaimi (1995), Stewart y Fenn (2006), } \\
\text { Kornish y Ulrich (2011) }\end{array}$ \\
\hline $\mathbf{P}_{10}$ & $\begin{array}{l}\text { La existencia de un sistema de gestión de la calidad } \\
\text { certificado según la norma ISO } 9001 \text { facilita la } \\
\text { implantación de un sistema de gestión de la innovación. }\end{array}$ & $\begin{array}{l}\text { Prajodo y Sohal (2006), Santos-Vijande } \\
\text { y Alvarez-Gonzalez (2007), Casadesús } \\
\text { et al. (2011) }\end{array}$ \\
\hline $\mathbf{P}_{11}$ & $\begin{array}{l}\text { La existencia de un sistema de gestión de la innovación } \\
\text { estimula la contratación de empresas especializadas } \\
\text { que aporten valor al proceso innovador. }\end{array}$ & Blayse y Manley (2004), Wagner (2006) \\
\hline $\mathbf{P}_{12}$ & $\begin{array}{l}\text { La implicación activa del jefe de obra en el proceso } \\
\text { innovador tiene un impacto significativo en los } \\
\text { resultados de la innovación. }\end{array}$ & Park et al. (2004), Dulaimi et al. (2005) \\
\hline $\mathbf{P}_{13}$ & $\begin{array}{l}\text { La innovación en la construcción requiere la } \\
\text { participación de equipos multidisciplinares. }\end{array}$ & Gann y Salter (2000), Bossink (2004) \\
\hline $\mathbf{P}_{14}$ & $\begin{array}{l}\text { La adopción de un sistema de gestión de la innovación } \\
\text { mejora la capacidad tecnológica de la empresa. }\end{array}$ & $\begin{array}{l}\text { Tatum (1987), Nam y Tatum (1992), } \\
\text { Slaughter (2000) }\end{array}$ \\
\hline $\mathbf{P}_{15}$ & $\begin{array}{l}\text { La adopción de un sistema de gestión de la innovación } \\
\text { incrementa la competitividad de la empresa. }\end{array}$ & $\begin{array}{l}\text { Tatum (1987), Nam y Tatum (1992), } \\
\text { Mitropoulus y Tatum (2000) }\end{array}$ \\
\hline $\mathbf{P}_{16}$ & $\begin{array}{l}\text { La certificación de un proyecto de innovación mejora } \\
\text { los resultados de la obra. }\end{array}$ & $\begin{array}{l}\text { Marimon y Cristobal (2005), Vea et al. } \\
\text { (2010) }\end{array}$ \\
\hline $\mathbf{P}_{17}$ & $\begin{array}{l}\text { La innovación en la construcción se frena cuando los } \\
\text { directivos de la empresa constructora no la perciben } \\
\text { como una estrategia competitiva. }\end{array}$ & $\begin{array}{l}\text { Nam y Tatum (1997), Slaughter (2000), } \\
\text { Blayse y Manley (2004) }\end{array}$ \\
\hline $\mathbf{P}_{18}$ & $\begin{array}{l}\text { La priorización de los procesos productivos dificulta la } \\
\text { detección de oportunidades para innovar. }\end{array}$ & $\begin{array}{l}\text { Tatum (1986), Pries y Janszen (1995), } \\
\text { Gann y Salter (2000) }\end{array}$ \\
\hline
\end{tabular}

Tabla 1. Proposiciones del estudio de caso y principales referencias que las respaldan (Pellicer et al. 2012) 


\begin{tabular}{cccc}
\hline Empresa & $\mathbf{N}^{\mathbf{0}}$ empleados & $\begin{array}{c}\text { Facturación (millones } \\
\text { de Euros) }\end{array}$ & $\begin{array}{c}\text { Inversión en innovación } \\
\text { (miles de Euros) }\end{array}$ \\
\hline Lambda & 430 & 488 & 200 \\
A & 3,100 & 2,600 & 18,000 \\
B & 2,200 & 900 & 4,000 \\
C & 7,000 & 2,700 & 18,500 \\
D & 15,000 & 3,500 & 1,500 \\
E & 80 & 60 & 5 \\
F & 500 & 190 & 300 \\
G & 500 & 150 & 2,000 \\
\hline
\end{tabular}

Tabla 2. Características básicas de las empresas (valores medios de 2007) 


\begin{tabular}{lcccccc}
\hline & $\mathbf{P}_{\mathbf{2}}$ & $\mathbf{P}_{\mathbf{4}}$ & $\mathbf{P}_{\mathbf{7}}$ & $\mathbf{P}_{\mathbf{1 5}}$ & $\mathbf{P}_{\mathbf{1 6}}$ & $\mathbf{P}_{\mathbf{1 7}}$ \\
\hline Empresas & $\mathbf{F}$ & $\mathrm{D}$ & $\mathbf{F}$ & $\mathrm{M}$ & $\mathrm{M}$ & $\mathbf{F}$ \\
Lambda & $\mathbf{F}$ & $D$ & $\mathbf{F}$ & $\mathrm{M}$ & $\mathrm{M}$ & $\mathbf{F}$ \\
Expertos & $\mathrm{M}$ & $\mathrm{M}$ & $\mathrm{M}$ & $\mathrm{M}$ & $\mathrm{D}$ & $\mathrm{M}$ \\
\hline MEDIA & $\mathbf{F}$ & $\mathrm{D}$ & $\mathbf{F}$ & $\mathrm{M}$ & $\mathrm{M}$ & $\mathbf{F}$ \\
\hline
\end{tabular}

Tabla 3. Discrepancias en el grado de validación de las proposiciones 